Garrote-Rojas, D., Jiménez-Fernández, S. \& Ritacco-Real, P. (2021). Factores Asociados a las Estrategias de Aprendizaje en Estudiantes Universitarios con y sin trabajo remunerado. Revista Electrónica Interuniversitaria de Formación del Profesorado, 24(2),81-96.

DOI: https://doi.org/10.6018/reifop.443551

\title{
Factores Asociados a las Estrategias de Aprendizaje en Estudiantes Universitarios con y sin trabajo remunerado
}

\author{
Daniel Garrote Rojas ${ }^{1}$, Sara Jiménez-Fernández ${ }^{2}$ y Paola Ritacco Real ${ }^{1}$ \\ 1 Universidad de Granada \\ 2 Unidad de Salud Mental Infanto -Juvenil del Complejo Hospitalario de Jaén
}

\section{Resumen}

El trabajo de investigación que a continuación se describe pretende dar a conocer las diferentes estrategias de aprendizaje utilizadas por los estudiantes universitarios para afrontar las asignaturas, así como, las diferencias que pudieran darse entre los estudiantes que a la vez que cursan las asignaturas están trabajando y los que no trabajan. La muestra tiene una edad promedio de 22 años, con una mayoría de sujetos femeninos $92 \%$ frente a los masculinos y pertenecen a los cursos académicos 2017/18 y 2018/19 en la Universidad de Castilla-La Mancha, utilizando el Cuestionario de Estrategias de Aprendizaje y Motivación (CEAM II). Los resultados muestran diferencias en cinco subescalas: elaboración, aprovechamiento del tiempo-concentración, ayuda, constancia y autointerrogación. Los estudiantes que compatibilizan su formación universitaria con un trabajo retribuido y los estudiantes sin trabajo retribuido. Obteniéndose mayores puntuaciones medias para los sujetos que trabajan en seis de las siete subescalas frente a los que no trabajan, que obtuvieron mayor puntuación media en la subescala ayuda.

\section{Palabras clave}

Estrategias de aprendizaje; elaboración; organización; EEES.

\section{Factors Associated with Learning Strategies in University Students with and without paid work}

\section{Abstract}

The research work that is described below aims to present the different learning strategies used by university students to face the subjects, as well as the differences that could occur 
between the students who, while they are studying the subjects, are working and those who don't work. The sample has an average age of 22 years, with a majority of female subjects 92\% compared to males and belong to the academic years 2017/18 and 2018/19 at the University of Castilla-La Mancha, using the Questionnaire on Strategies of Learning and Motivation (CEAM II). The results show differences in five subscales: elaboration, use of timeconcentration, help, perseverance and self-examination. Students who combine their university education with paid work and students without paid work. Obtaining higher mean scores for the subjects who work in six of the seven subscales compared to those who don't work, who obtained a higher average score in the help subscale.

\section{Key words}

Learning strategies; elaboration; organization; EHEA.

\section{Introducción}

Tradicionalmente, se ha hecho referencia a los estilos de aprendizaje como variables personales para dar respuesta a cómo se aborda, planifica y responde a las demandas de aprendizaje. Según la teoría del aprendizaje experimental (Kolb, 1984) y su desarrollo, podemos clasificar las diferentes preferencias individuales de acceso al conocimiento por medio de cuatro estilos de aprendizaje (Camero, Martín y Herrero, 2000):

- Estilo activo, centrado en la vivencia directa.

- Estilo reflexivo, centrado en observar y recoger información.

- Estilo teórico, centrado en los conceptos abstractos y la formación de conclusiones.

- Estilo pragmático, basado en la experimentación activa y búsqueda de aplicaciones prácticas.

Las estrategias para adquirir el conocimiento se realizan mediante cuatro fases: la adquisición, la codificación, la recuperación y el apoyo para procesar los conceptos. Los modelos de formación del Espacio Europeo de Educación Superior (EEES) que pretenden modificar el entorno formal y la respuesta a la enseñanza universitaria, lleva a crear un nuevo modelo para el docente y discente (Malbernat, 2008; Rodríguez y Herrera, 2009; Fraile, López-Pastor, Castejón y Romero, 2013). Donde el alumno es el responsable de su proyecto formativo y se produce una innovación incesante en el sistema de formación. Aparecen nuevas titulaciones comunes para las universidades europeas (Cuadrado y Fernández, 2008; Goñi, 2005; Herrera, Lorenzo y Rodríguez, 2008; Malbernat, 2008; Tomusk, 2006; Zabalza, 2003). Resaltando los objetivos alcanzados en el aprendizaje, lo que el alumno puede lograr cuando finalice su proceso formativo y la metodología que le facilitará seguir adquiriendo conocimiento autónomamente (Delgado, 2006; Hernández-Pina, Clares, Rosario y Espin, 2005; Moreno, Bajo, Moya, Maldonado y Tudela, 2007; Oliveros, 2006; Malbernat, 2008). A su vez es importante disponer de mecanismos de control de calidad para las instituciones educacionales, donde los resultados de aprendizajes anticipados podrán responsabilizarse de las necesidades y retos a los que tienen que hacer frente los universitarios en su proceso de educación (Cano, Chamizo y Valiente, 2014).

Durante el proceso de enseñanza-aprendizaje, el docente debe guiar y ayudar al alumno a aprender, adaptándose a sus necesidades de forma comprensiva (Black y Wiliam, 1998; Brookhart, 2007; Yorke, 2003) y ser capaz de transmitir esa energía activa que estimule al alumnado (Palomares y Garrote, 2009), así como adaptarse a los tiempo actuales buscando 
metodologías más participativas y activas (Abellán y Herrada, 2016). Un adecuado uso del feedback durante la evaluación formativa demuestra mejoras durante el aprendizaje y los resultados de los estudiantes (Ferguson, 2011; Nicol y Macfarlane, 2006; Sadler, 2010). Para poder examinar este nuevo proceso de formación de los estudiantes hay que alcanzar un mejor rendimiento en sus capacidades en comparación con otros procesos formativos (Rodríguez y Herrera, 2009; Fraile, López-Pastor, Castejón y Romero, 2013).

Pintrich et al., (1988) "revisaron diferentes cuestionarios asociados a los procesos internos que utilizan los estudiantes (cognitivos y/o motivacionales), así como variables cognitivas, motivacionales y los resultados en el rendimiento académico o en el desempeño de tareas específicas". Las estrategias y la motivación se relacionan positivamente, alcanzándose un buen rendimiento durante su formación por parte de los estudiantes (Pintrich y DeGroot, 1990; Martínez-Fernández y Galán, 2000, p37).

Mediante el CEAM II (Roces, Tourón y González, 1995; Martínez-Fernández y Galán, 2000, p.38; Rodríguez y Herrera, 2009), "se procura configurar un punto de vista cognitivo en la motivación y en las estrategias de aprendizaje de los estudiantes. Procede de la versión y adecuación del Motivated Strategies for Learning Questionnaire (MSLQ), creado por Pintrich et al., 1988, 1993, diseñado para evaluar las orientaciones motivacionales de los estudiantes y qué estrategias de aprendizaje emplean a lo largo de un curso académico". Respecto a las estrategias, hay tres aspectos significativos a destacar: las estrategias metacognitivas que sirven para planificar la formación, encauzarla y adaptarla a los procesos cognitivos, administrar adecuadamente los recursos del estudiante y la organización cognitiva del sujeto para entender, asimilar y evocar el conocimiento asimilado en las diferentes asignaturas que completan su formación (Printrich y DeGroot, 1990; Roces, Tourón y González, 1995). El cuestionario MSLQ ha servido para describir entornos universitarios (Roces, Tourón y González, 1995; Garrote, Garrote y Jiménez, 2016) y no universitario, llevándose a cabo análisis estadísticos en los resultados académicos obtenidos. Respecto al trabajo realizado por Roces, Tourón y González (1995) sobre la adaptación y traducción al castellano del cuestionario, han obtenido dos versiones del CEAM: CEAM I y II.

\section{Objetivos}

A través de esta investigación buscamos conocer qué estrategias seleccionan los estudiantes para afrontar su formación universitaria. Como objetivos específicos:

- Identificar los factores que se asocian a las estrategias utilizadas por los estudiantes.

- Describir el valor que le otorgan los estudiantes a cada factor.

- Analizar la relación que pudiese darse entre las estrategias de aprendizaje llevadas a cabo por aquellos estudiantes que además de desarrollar su tarea formativa en el Grado están trabajando y los estudiantes que no trabajan.

Estos objetivos obedecen al interés como docentes universitarios por optimizar los recursos de los estudiantes durante su formación.

\section{Metodología}

Analizando la realidad social y económica actual de los estudiantes universitarios, se ha decidido explicar el efecto que tiene desarrollar un trabajo retribuido o no simultáneamente al estudio de un Grado universitario. Se ha llevado a cabo una metodología descriptiva de corte transversal desde una perspectiva cuantitativa (Vázquez y Sevillano, 2015). La 
obtención de las respuestas a los cuestionario se realizó en el aula, durante el último mes de finalización del curso, previo a los exámenes.

\section{Participantes}

Participaron 675 universitarios de los Grados de Maestro en la Universidad de Castilla-La Mancha durante dos cursos académicos (2017/18 y 2018/19). Los estudiantes participantes del sexo femenino supuso el $92 \%$ de la muestra total, con una edad media de 22 años. La muestra se seleccionó mediante contacto directo con los estudiantes de primer y cuarto curso.

\section{Instrumentos/Materiales}

Para poder analizar las estrategias de aprendizaje utilizas los estudiantes, aplicaremos el cuestionario CEAM II (Roces, Tourón y González, 1995, p.351), "el cual ofrece una visión sobre motivación y estrategias de aprendizaje de los estudiantes. Respecto a las estrategias de aprendizaje, esta está formada por las siguientes subescalas: Elaboración, Aprovechamiento del tiempo-concentración, Organización, Ayuda, Constancia, Metacognición y Autointerrogación". En cuanto a los índices de fiabilidad (de Cronbach) del conjunto del cuestionario, alcanza un 0.89 , la fiabilidad de las escalas que hacen referencia a las estrategias de aprendizaje oscilan entre 0.68 y 0.84 .

El cuestionario CEAM II, está formado por 81 ítems, 31 se refieren a la motivación y 50 a las estrategias de aprendizaje. El tipo de respuesta empleado es tipo Likert de 7 puntos, relacionado con el nivel de acuerdo de cada uno de los ítems.

\section{Análisis de datos}

El análisis de datos se ha llevado a cabo con el Statistical Package for the Social Science (SPSS) versión 22.0. Haciendo un análisis descriptivo, para analizar el valor que alcanzan los universitarios en los diferentes factores sobre estrategias de aprendizaje. Comprobando que no siguen las variables una distribución normal (Test de Kolmogorov-Smirnov), se realizó un análisis de medias independientes (ANOVA) de las diferentes subescalas de las estrategias de aprendizaje entre los alumnos con y sin trabajo. El nivel de significación que se estableció fue de $p<0.05$.

\section{Resultados}

Los estudiantes otorgan una mayor puntuación total al empleo de dos estrategias de aprendizaje: la Elaboración (3594 puntos y una media de 5.32) y la Organización (3475 puntos y una media de 5.15). La primera valora si los estudiantes utilizan conocimientos previos en situaciones nuevas para dar solución a los problemas, tomar decisiones, y si establecen conexiones de la nueva información con la que tenían anteriormente. La Organización incide en las estrategias llevadas a cabo por los universitarios para adquirir el contenido y escoger la información mediante técnicas como realizar esquematizar, resumir o subrayar. Respecto a las estrategias que obtienen una menor puntuación y por tanto, menos utilizadas son: Constancia (2911 puntos y una media de 4.45), se refiere al empeño y celeridad para llevar las actividades al día y lograr así las metas establecidas. Aprovechamiento del tiempoConcentración (3026 puntos y una media de 4.48), indica la condición del estudiante para concentración en la actividad que tiene que realizar y la capacidad de aprovechar su tiempo de estudio. Autointerrogación (3039 puntos y una media de 4.50) hace referencia a las cuestiones que se hace el estudiante durante el aprendizaje con el objetivo de centrarse en la materia, autoevaluar su nivel de comprensión y conocer qué ha aprendido realmente. Las 
puntuaciones intermedias se obtuvieron en los factores de Metacognicción (3391 puntos y una media de 4.66) y Ayuda (3145 puntos y una media de 5.02).

En la tabla 1 observamos las medias, desviaciones típicas y la medianas que se obtienen en los ítems que forman cada una de las subescalas de las estrategias de aprendizaje. Los ítems a los que los sujetos han otorgado mayor puntuación, de forma creciente, en primer lugar el ítem 73: "Asisto a clase regularmente", con una puntuación global de 4554 puntos, una media de 6.75 y una mediana de 7, pertenece al factor constancia. Posteriormente el ítem 35: "Usualmente estudio en un lugar donde pueda concentrarme", con una puntuación global de 4347 puntos, una media de 6.44 y una mediana de 7 . Incluido en el factor aprovechamiento, que muestra la capacidad del estudiante para aprovechar y gestionar el tiempo invertido en su formación y la habilidad para no distraerse en esa actividad. Ítems 41: "Cuando estoy confundido(a) acerca de algo que estoy leyendo para esta asignatura, vuelvo a leerlo y trato de entenderlo", con una puntuación global de 4221 puntos, una media de 6.25 y una mediana de 7. Situado en el factor metacognición y dirigido a establecer metas, la ordenación del aprendizaje y al entendimiento. Ítem 63: "Cuando estudio para esta asignatura, voy a mis apuntes y subrayo los conceptos importantes", con una puntuación global de 4044 puntos, una media de 5.00 y una mediana de 6. Incluido en el factor de organización, indica las tácticas empleadas por los estudiantes para adquirir conocimientos y qué método utilizan para seleccionar la información. Ítem 64: "Cuando leo para esta asignatura trato de relacionar el material con el que ya conozco", con una puntuación global de 4054 puntos, una media de 6.01 y una mediana de 6. Pertenece al factor elaboración, que considera si el estudiante utiliza conocimientos anteriores a una situación nueva para obtener una respuesta a los problemas y si realiza relaciones entre la información que ya tenía y la nueva.

Con respecto a los ítems con menor valoración por parte de los estudiantes son: ítem 37: "Frecuentemente me siento tan perezoso(a) o aburrido(a) cuando estudio para esta asignatura que abandono el estudio antes de finalizar lo que planeaba hacer", con una puntuación global de 1186 puntos, una media de 1.76 y una mediana de 1. Está asociado a la constancia, muestra el esfuerzo que deben realizar los universitarios en las actividades para alcanzar sus metas. Ítem 60: "Cuando el material y/o las tareas son difíciles, las abandono y sólo estudio las partes fáciles", con una puntuación global de 1209 puntos, una media de 1.79 y una mediana de 1. Incluido en el factor constancia, indica la percepción de los estudiantes en lograr sus objetivos. Ítem 57: "Frecuentemente me doy cuenta de que no he comprendido bien lo que he leído para esta asignatura", con una puntuación global de 1702 puntos, una media de 2.52 y una mediana de 2. Pertenece al factor metacognición. Tanto el ítem 33: "Durante las clase con frecuencia se me escapan puntos importantes, porque estoy pensando en otras cosas", con una puntuación global de 1853 puntos, una media de 2.75 y una mediana de 2, como el ítem 52: "Se me hace difícil sujetarme a un horario de estudio", con una puntuación global de 2185 puntos, una media de 3.24 y una mediana de 3, están incluidos en el factor aprovechamiento del tiempo-concentración. La correcta metodología de la asignaturas y cómo se llevan a cabo las actividades, no es un elemento que destaque.

Uno de los datos que se solicitó a la muestra fue que indicasen si estaban realizando algún trabajo retribuido o no mientras llevaban a cabo sus estudios universitarios. Teniendo en cuenta los sujetos que llevan a cabo una tarea retributiva durante su formación académica (trabajan), y quienes no la tienen (no trabajan). En la tabla 2 se indican los valores de comparar ambos grupos. 
Tabla 1.

Puntuaciones de los ítems en las subescalas de las estrategias de aprendizaje

\begin{tabular}{|c|c|c|c|c|}
\hline Subescalas & Ítems & Media & $\begin{array}{l}\text { Desviación } \\
\text { típica }\end{array}$ & Mediana \\
\hline \multirow{8}{*}{$\begin{array}{l}: \frac{0}{U} \\
: \frac{0}{0} \\
\frac{0}{0} \\
\frac{0}{w}\end{array}$} & 47 & 4.43 & 1.663 & 5 \\
\hline & 51 & 4.75 & 1.458 & 5 \\
\hline & 62 & 5.76 & 1.141 & 6 \\
\hline & 64 & 6.01 & 1.058 & 6 \\
\hline & 66 & 5.63 & 1.058 & 6 \\
\hline & 69 & 5.76 & 1.045 & 6 \\
\hline & 71 & 4.76 & 1.276 & 5 \\
\hline & 81 & 5.50 & 1.452 & 6 \\
\hline \multirow{6}{*}{ 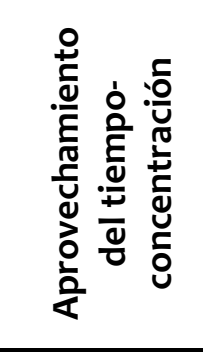 } & 33 & 2.75 & 1.687 & 2 \\
\hline & 35 & 6.44 & 0.931 & 7 \\
\hline & 43 & 5.28 & 1.321 & 5 \\
\hline & 52 & 3.24 & 1.970 & 3 \\
\hline & 65 & 6.20 & 1.370 & 7 \\
\hline & 77 & 2.99 & 1.672 & 3 \\
\hline \multirow{8}{*}{ 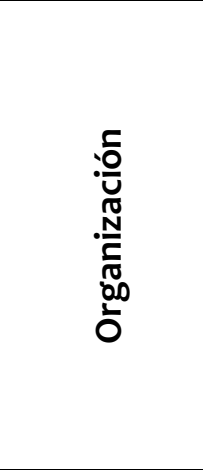 } & 32 & 5.67 & 1.439 & 6 \\
\hline & 42 & 5.92 & 1.045 & 6 \\
\hline & 49 & 3.52 & 1.982 & 3 \\
\hline & 53 & 5.08 & 1.435 & 5 \\
\hline & 59 & 5.37 & 1.456 & 6 \\
\hline & 63 & 5.99 & 1.055 & 6 \\
\hline & 67 & 5.61 & 1.455 & 6 \\
\hline & 72 & 4.02 & 1.732 & 4 \\
\hline \multirow{7}{*}{$\frac{\pi}{\tilde{z}}$} & 34 & 4.18 & 1.785 & 5 \\
\hline & 40 & 4.32 & 1.757 & 5 \\
\hline & 45 & 5.17 & 1.478 & 6 \\
\hline & 50 & 3.51 & 1.804 & 3 \\
\hline & 58 & 4.93 & 1.603 & 5 \\
\hline & 68 & 5.50 & 1.423 & 6 \\
\hline & 75 & 5.00 & 1.695 & 5 \\
\hline \multirow{7}{*}{ 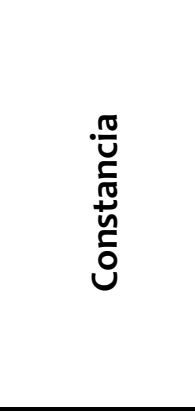 } & 37 & 1.76 & 1.192 & 1 \\
\hline & 39 & 4.26 & 1.927 & 5 \\
\hline & 46 & 5.46 & 1.343 & 6 \\
\hline & 48 & 4.97 & 1.912 & 6 \\
\hline & 60 & 1.79 & 1.204 & 1 \\
\hline & 70 & 5.20 & 1.479 & 6 \\
\hline & 73 & 6.75 & 0.708 & 7 \\
\hline
\end{tabular}




\begin{tabular}{|c|c|c|c|c|}
\hline \multirow{7}{*}{ 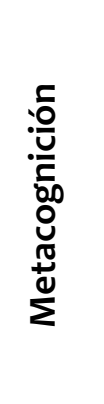 } & 41 & 6.25 & 0.951 & 6 \\
\hline & 44 & 5.09 & 1.391 & 5 \\
\hline & 54 & 5.70 & 1.322 & 6 \\
\hline & 56 & 5.15 & 1.332 & 5 \\
\hline & 57 & 2.52 & 1.534 & 2 \\
\hline & 76 & 5.63 & 1.134 & 6 \\
\hline & 78 & 4.83 & 1.594 & 5 \\
\hline \multirow{4}{*}{ 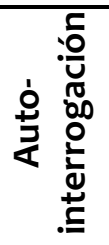 } & 36 & 4.02 & 1.877 & 4 \\
\hline & 38 & 4.24 & 1.550 & 4 \\
\hline & 55 & 4.97 & 1.628 & 5 \\
\hline & 61 & 4.78 & 1.599 & 5 \\
\hline
\end{tabular}

Tabla 2.

Análisis de las subescalas y los ítems de la estrategias de aprendizaje según tengan trabajo retribuido o no

\begin{tabular}{|c|c|c|c|c|c|}
\hline Subescalas & & $\begin{array}{l}\text { Trabajan } \\
(n=99)\end{array}$ & $\begin{array}{l}\text { No } \\
\text { trabajan } \\
(n=576)\end{array}$ & $\begin{array}{l}\text { Valor de } \\
p\end{array}$ & z \\
\hline \multirow{2}{*}{ Elaboración } & Media & $5.57 \pm 0.82$ & $5.28 \pm 0.8$ & \multirow{2}{*}{0.001} & \multirow{2}{*}{-3.295} \\
\hline & Mediana & 5.63 & 5.38 & & \\
\hline \multirow{2}{*}{$\begin{array}{l}\text { Aprovechamiento } \\
\text { del tiempo- } \\
\text { concentración }\end{array}$} & Media & $4.63 \pm 0.72$ & $4.46 \pm 0.65$ & \multirow[t]{2}{*}{0.035} & \multirow[t]{2}{*}{-2.108} \\
\hline & Mediana & 4.67 & 4.33 & & \\
\hline \multirow{2}{*}{ Organización } & Media & $5.15 \pm 0.51$ & $5.14 \pm 0.82$ & \multirow{2}{*}{0.705} & \multirow{2}{*}{-0.378} \\
\hline & Mediana & 5.13 & 5.19 & & \\
\hline \multirow{2}{*}{ Ayuda } & Media & $5.04 \pm 1.27$ & $5.44 \pm 0.88$ & \multirow{2}{*}{0.001} & \multirow{2}{*}{-4.039} \\
\hline & Mediana & 4.43 & 4.71 & & \\
\hline \multirow{2}{*}{ Constancia } & Media & $6.07 \pm 0.59$ & $6.02 \pm 0.68$ & \multirow{2}{*}{0.001} & \multirow{2}{*}{-4.267} \\
\hline & Mediana & 4.25 & 4.50 & & \\
\hline \multirow{2}{*}{ Metacognición } & Media & $5.41 \pm .071$ & $5.32 \pm 0.74$ & \multirow{2}{*}{0.528} & \multirow{2}{*}{-0.631} \\
\hline & Mediana & 5.14 & 5.14 & & \\
\hline \multirow{2}{*}{$\begin{array}{l}\text { Auto- } \\
\text { interrogación }\end{array}$} & Media & $3.18 \pm 0.94$ & $3.12 \pm 0.86$ & \multirow{2}{*}{0.001} & \multirow{2}{*}{-4.148} \\
\hline & Mediana & 4.75 & 4.50 & & \\
\hline
\end{tabular}

Los alumnos sin trabajo representan el $85.33 \%$ de la muestra y los que trabajan el $14.67 \%$. De la tabla 2 se desprende la relevancia de las subescalas Elaboración, Aprovechamiento del 
tiempo-concentración, Ayuda, Constancia y Autointerrogación. En la Elaboración se ha obtenido un valor de $p$ de 0.001 al comparar las medias que obtienen los estudiantes que trabajan $(5.57 \pm 0.82)$ y los estudiantes que no trabajan $(5.28 \pm 0.8)$. En la subescala Aprovechamiento del tiempo-concentración se ha obtenido un valor de $p$ de 0.035 entre los estudiantes con trabajo $(4.63 \pm 0.72)$ y sin trabajo $(4.46 \pm 0.65)$. Para la subescala Ayuda el valor de $p$ ha sido de 0.001 entre los estudiantes con trabajo $(5.04 \pm 1.27)$ y sin trabajo $(5.44 \pm$ 0.88). En la subescala Constancia el valor de $p$ ha sido de 0.001 para los estudiantes con trabajo $(6.07 \pm 0.59)$ y los estudiantes que sin trabajo $(6.02 \pm 0.68)$. Por último, para la subescala Autointerrogación el valor de $p$ ha sido 0.001 entre los estudiantes que trabajan (3.18 \pm 0.94$)$ y los que no trabajan (3.12 \pm 0.86$)$.

En el factor Elaboración (figura 1), ambas muestras obtuvieron mayoritariamente las puntuaciones entre los intervalos de respuesta 5: "Me describe suficientemente" y 6: "Me describe mucho". Los sujetos que no trabajaban, el porcentaje mayor de la muestra, respondió entre los intervalos de respuesta 4 y 6 , mientras que de los sujetos que están trabajando respondieron entre el 5 y el 6 . Los ítems que obtienen mayor puntuación son el ítem 64: "Cuando leo para esta asignatura trato de relacionar el material con el que ya conozco" y el ítem 69: "Trato de entender bien el material de esta asignatura para hacer conexiones entre las lecturas y los conceptos estudiados".

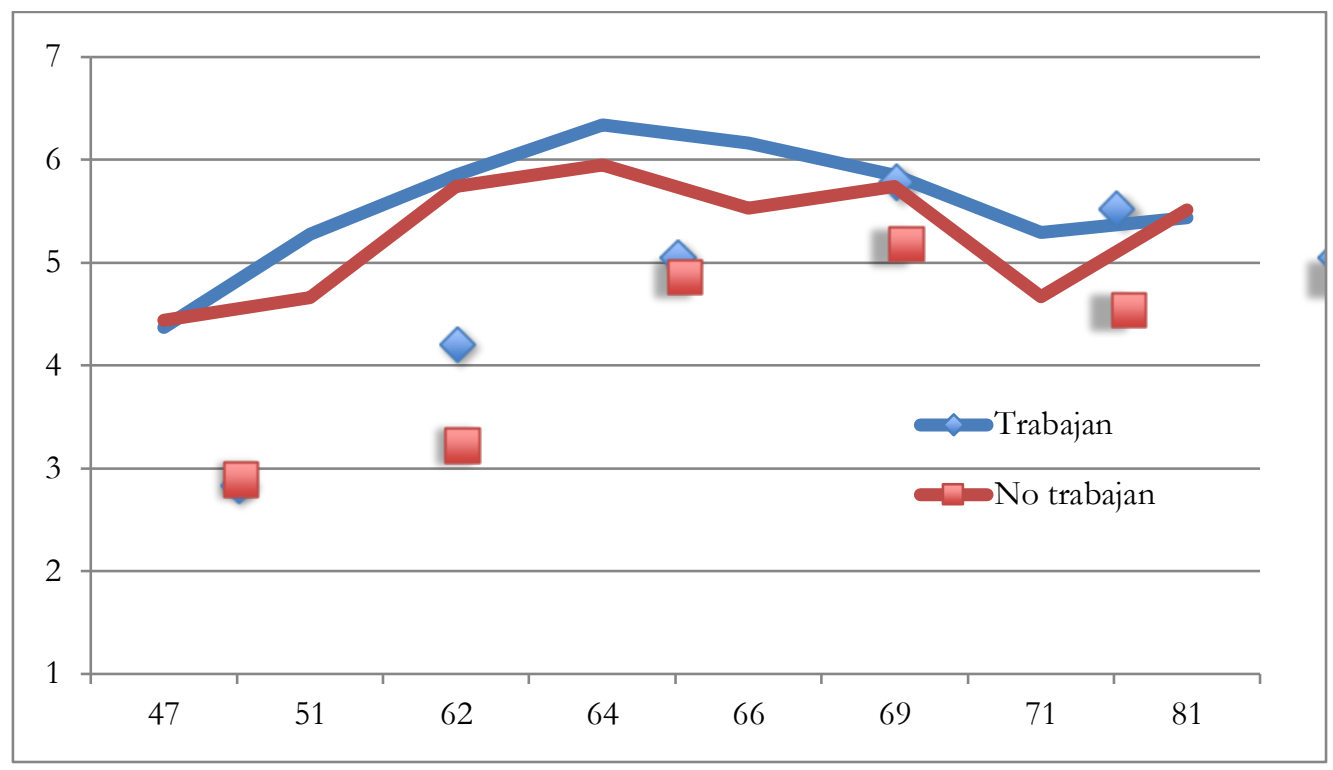

Figura 1. Factor Elaboración

Respecto al factor Metacognición (figura 2), la muestra de los sujetos que trabajan respondieron entre los intervalos de respuesta 5 y 6 , siendo en el intervalo 5 por el que se decantó la mayoría de sujetos (63.64\%). Las respuesta de ambos grupos de sujetos fue similar. Destacan dos ítems de los que forma el factor, el ítem 41: "Cuando estoy confundido acerca de algo que estoy leyendo para esta asignatura, vuelvo a leerlo y trato de entenderlo", y el ítem 57: "Frecuentemente me doy cuenta de que no he comprendido bien lo que he leído para esta asignatura.", con puntuaciones de 1,88 para los estudiantes con trabajo y 2,63 para los que sin trabajo. En este ítem es donde obtienen mayor diferencia de respuesta. 


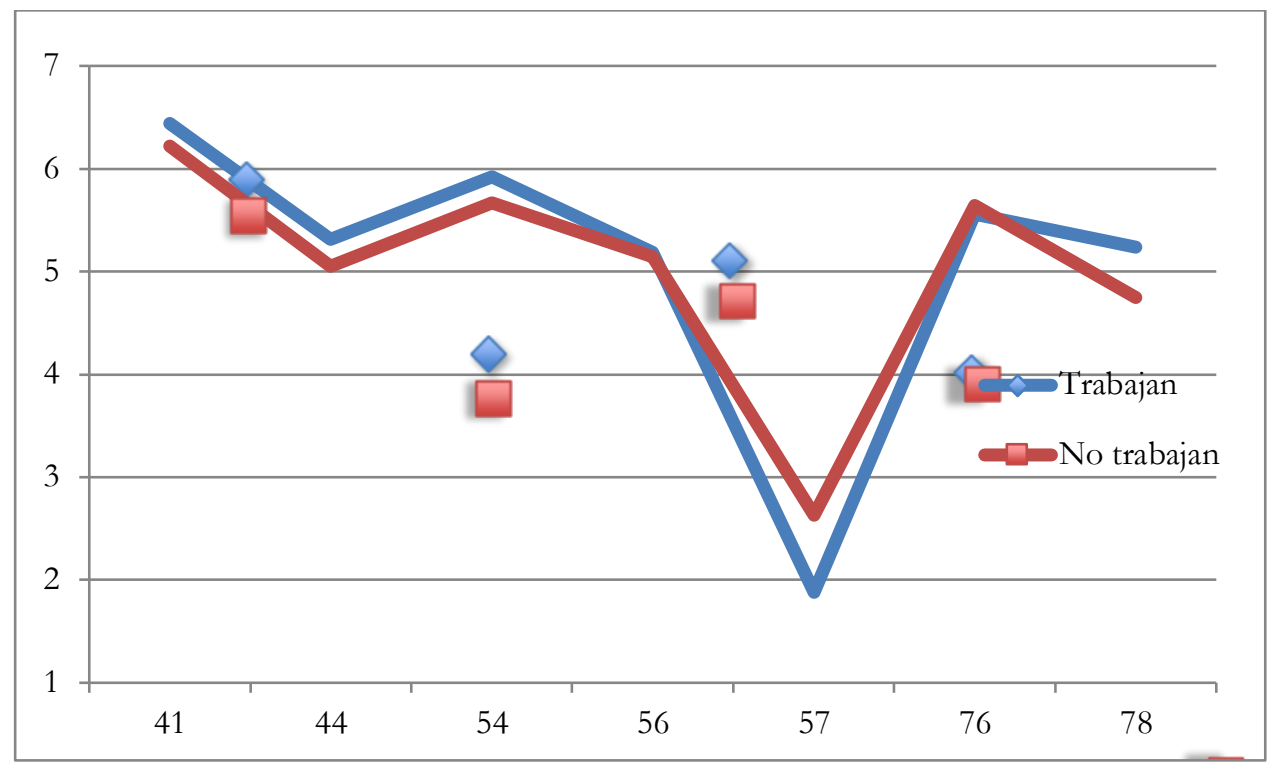

Figura 2. Factor Metacognición

En la figura 3, factor Organización, los estudiantes que trabajan respondieron entre el intervalo 4 y 6 , alcanzando la escala 5 la mayor frecuencia (59.09\%). Sin embargo, respecto a los sujetos que no trabajan utilizaron un intervalo mayor de respuestas, del 2 al 6 . Destacan dentro de los ítems más valorados, por ambos grupos, el ítems 63: "Cuando estudio para esta asignatura, voy a mis apuntes y subrayo los conceptos importantes" y el ítems 42: "Cuando estudio para esta asignatura me baso en las lecturas y trato de encontrar las ideas más importantes".

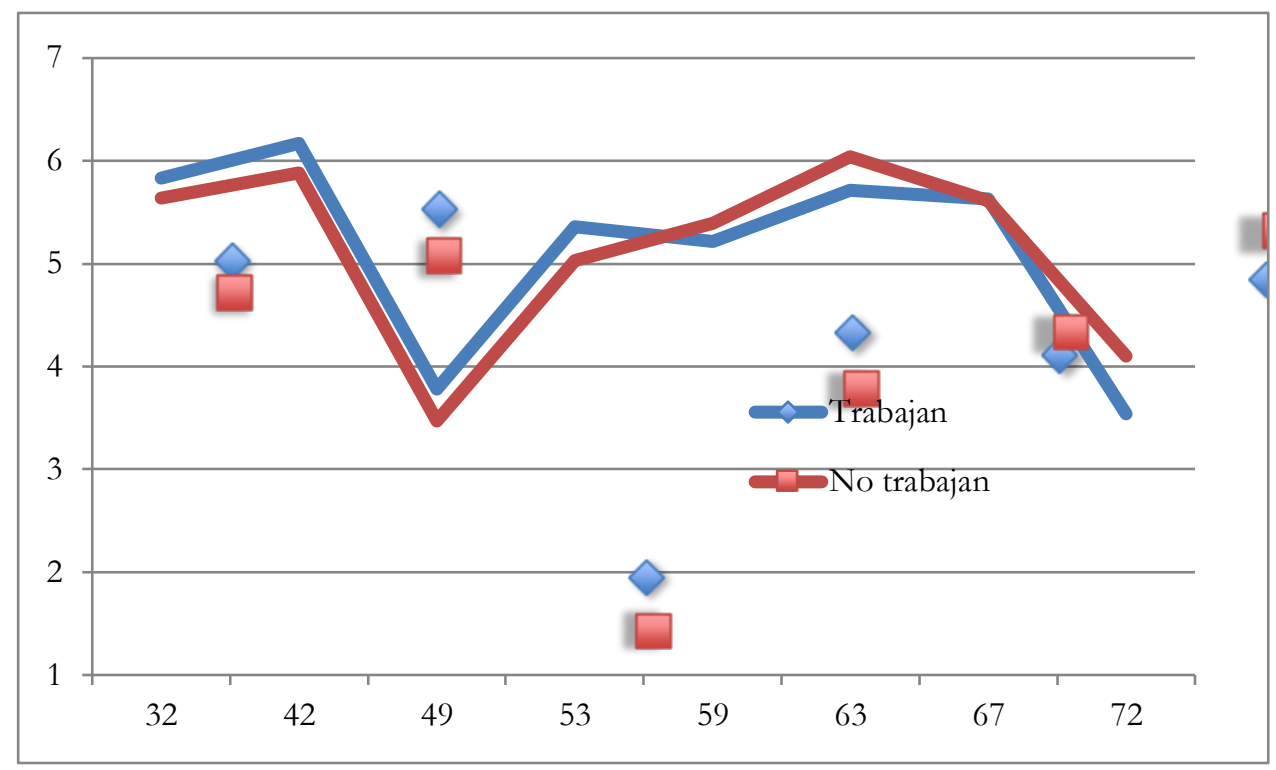

Figura 3. Factor Organización

En el factor Ayuda (figura 4), obtienen similar puntuación ambos grupos de sujetos, con el ligero matiz de que los que trabajan oscilan entre el intervalo 3 y $4, y$ los que no trabajan entre el 4 y 5 . Hay dos ítems que obtienen mayor relevancia, el ítem 68: "Cuando no puedo entender la asignatura pido a otro estudiante de la clase que me ayude" y el ítem 45: "Trato de trabajar con otros estudiantes de esta asignatura para completar las tareas asignadas". 


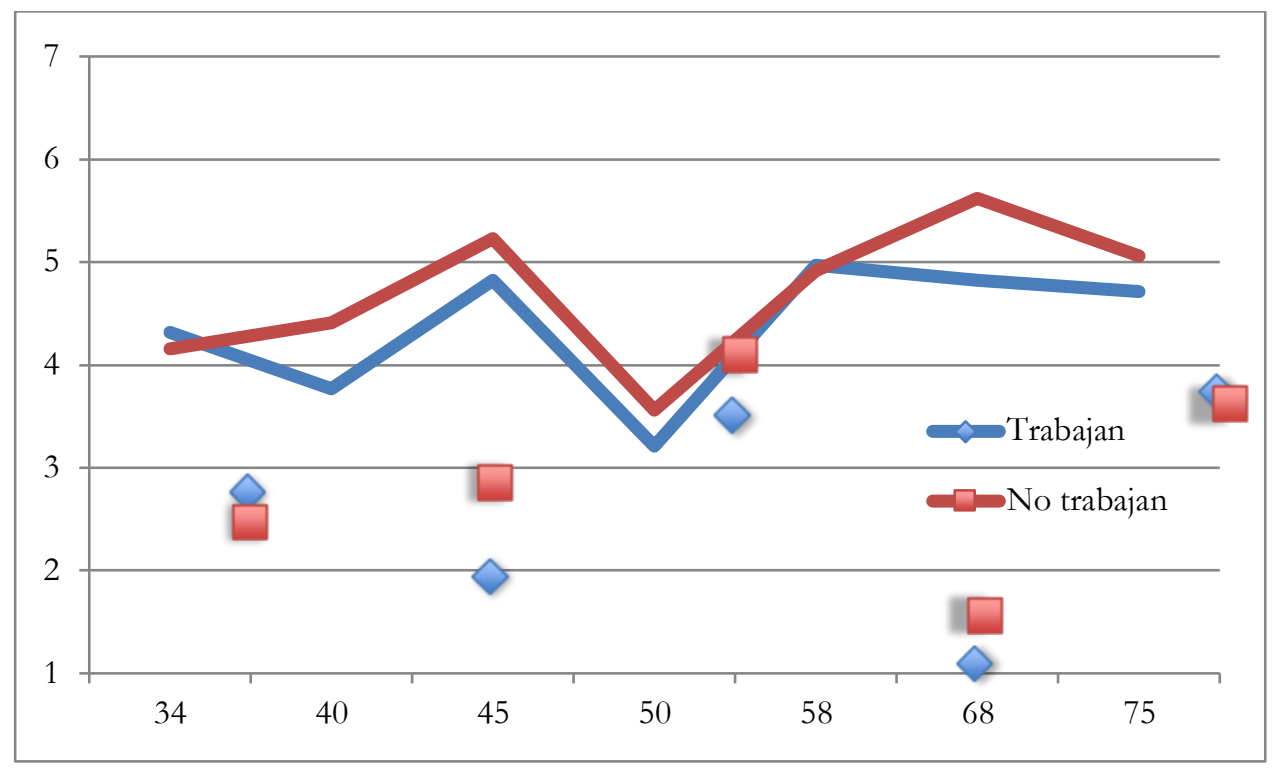

Figura 4. Factor Ayuda

Para el factor Constancia (figura 5), el reparto en el porcentaje de los sujetos que trabajan, se realiza mayoritariamente en el intervalo 3 y 4 , mientras que los sujetos que no trabajan utilizan el 4 y 5 . Los ítems con mayor puntuación son, el ítem 73: "Asisto a clase regularmente" y el ítem 46: "Cuando estudio para esta asignatura, leo las notas tomadas en clase y las lecturas una y otra vez".

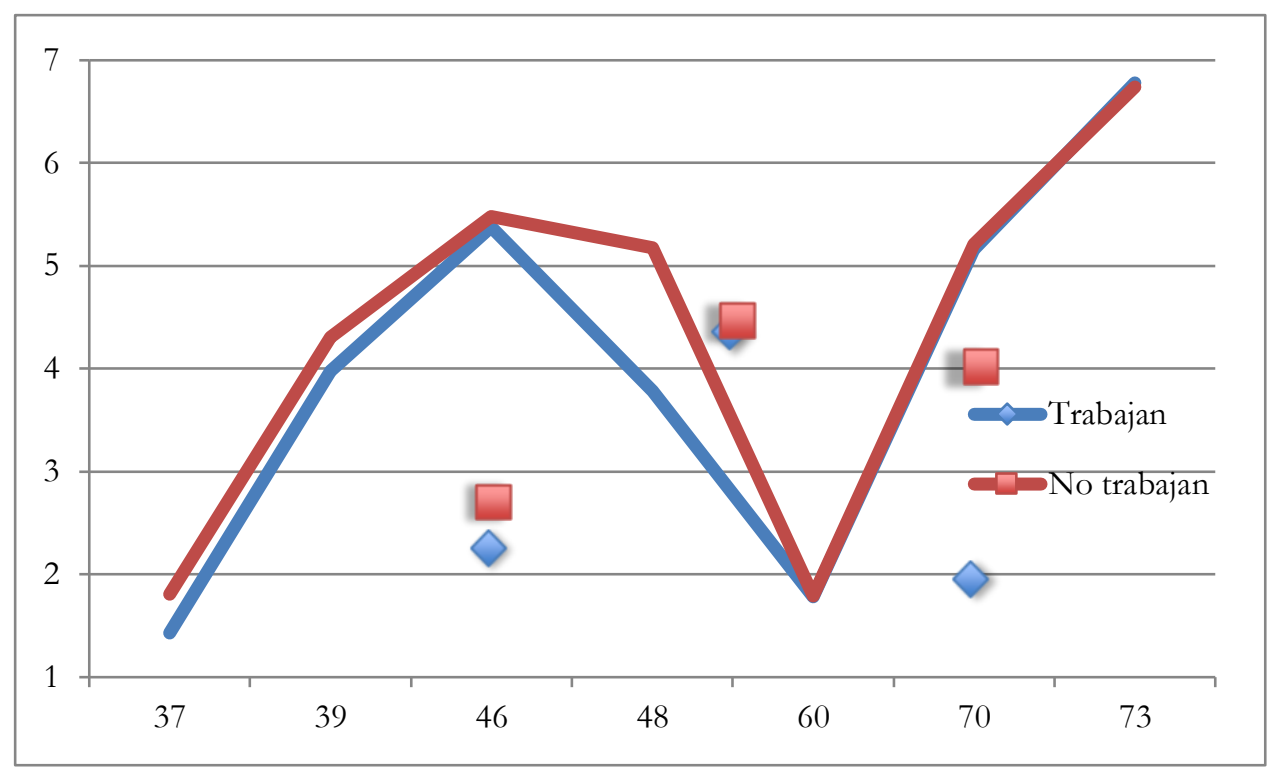

Figura 5. Factor Constancia

En el factor Aprovechamiento del tiempo-Concentración (figura 6), es similar el intervalo mayoritario de sujetos que trabajan y no trabajan, eligiendo las respuestas 3,4 y 5 . Los ítems 
más relevantes son, ítem 35: "Usualmente estudio en un lugar donde pueda concentrarme" y el ítem 65: "Tengo un espacio privado para estudiar".

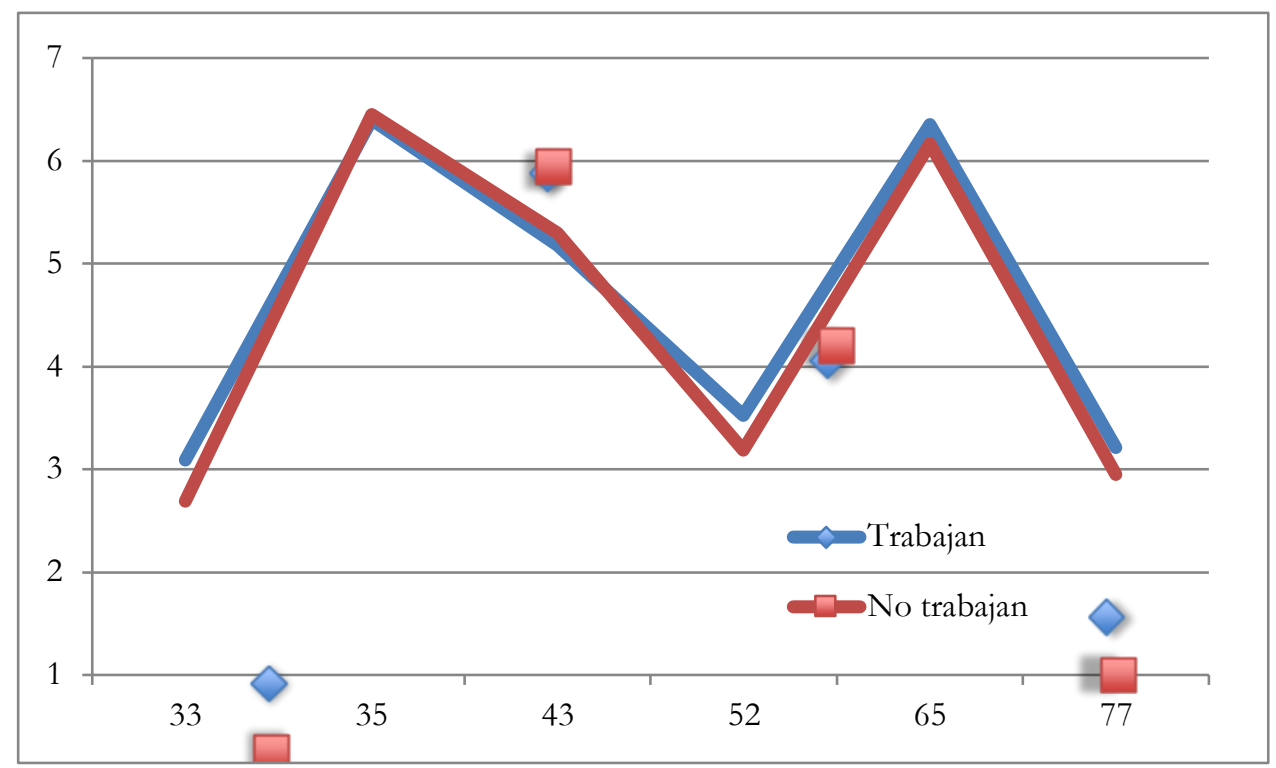

Figura 6. Factor Aprovechamiento-Concentración

Por último, la figura 7, el factor Autointerrogación, el grupo de sujetos que trabajan obtienen una mayor valoración en todos los ítems que el grupo de sujetos que no trabajan. Los ítems con mayores puntuaciones son el ítem 55: "Yo mismo me hago preguntas para asegurarme que entiendo el material que he estado estudiando en esta asignatura" y el ítem 61: "Trato de pensar sobre un tópico para decidir qué se supone que debo aprender sobre él, más que solamente leerlo y aprenderlo de memoria".

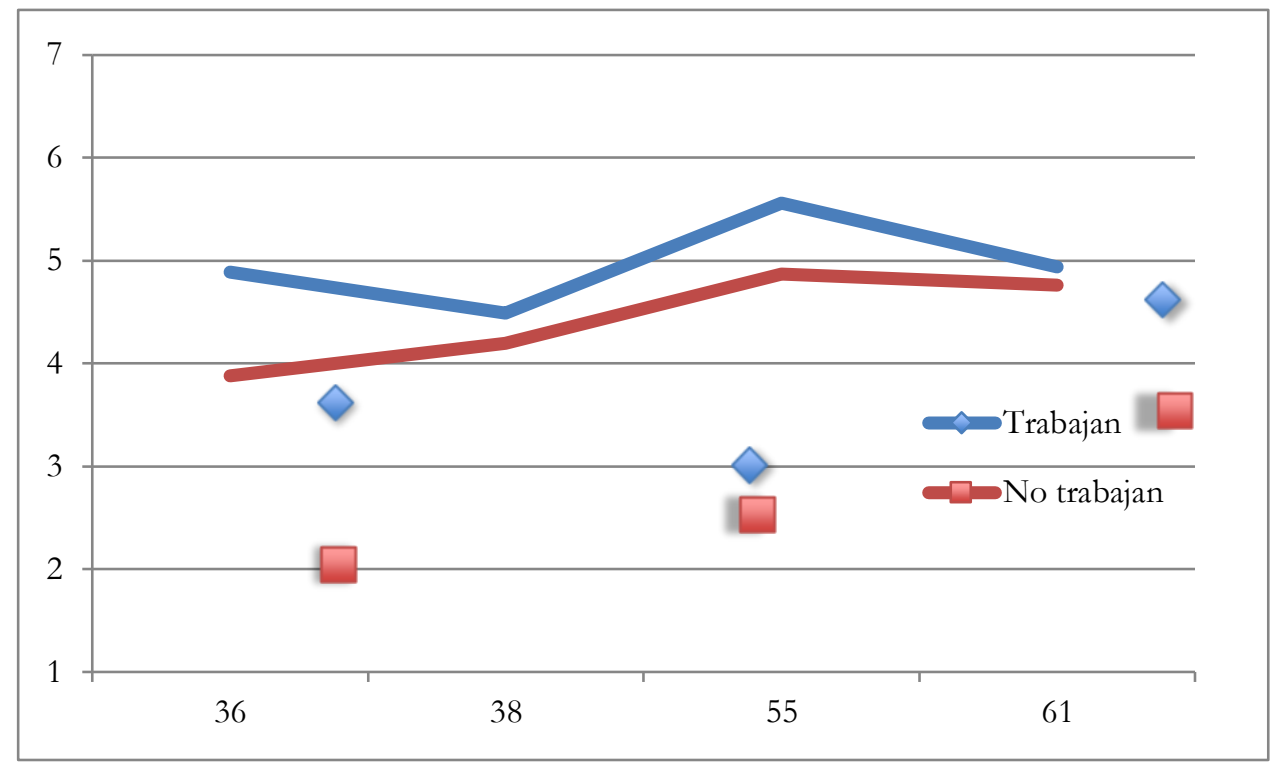

Figura 7. Autointerrogación 


\section{Discusión y conclusiones}

Destacando la repercusión que tiene para los estudiantes las estrategias que utilizan, se han analizado los datos obtenidos y los ítems con una mayor puntuación y por tanto un mayor efecto en las estrategias llevadas a cabo, haciendo referencia a los factores de Constancia, Aprovechamiento de tiempo-concentración, Metacognición, Organización y Elaboración. La asistencia a clase, el uso de un espacio donde pueda reflexionar y sedimentar los conocimientos, utilizar estrategias como el esquema o el subrayado y vincular la información nueva con el conocimiento previo de la materia mejorará el rendimiento académico. Mientras que los ítems con menor puntuación pertenecen a los factores constancia, metacognición y aprovechamiento.

Las puntuaciones totales obtenidas en los factores que forman las estrategias de aprendizaje, en orden decreciente su asignación ha sido: Elaboración (3594 puntos), Organización (3475 puntos), Metacognición (3391 puntos), Ayuda (3145 puntos), Autointerrogación (3039 puntos), Aprovechamiento del tiempo-concentración (3026 puntos) y Constancia (2911 puntos). La Elaboración, indica como el estudiante utiliza sus conocimientos previos en circunstancias nuevas para así ser capar de ofrecer una respuesta a las adversidades que le surgen. Al igual que indica Boza y Toscana (2012), entre las estrategias más usadas por los estudiantes se encuentran la capacidad del estudiante para distinguir los contenidos relevantes, las anotaciones y el uso de conocimientos previos (Boza y Méndez, 2013). La Organización muestra la habilidad que tiene el estudiante para integrar el contenido nuevo y diferenciar la información relevante utilizando herramientas como el esquema o el resumen. El uso de estrategias de aprendizaje de alto nivel con un alto nivel de organización se asocian al gusto por aprender y tareas abiertas (Monereo y Badia, 2013), así como dar respuesta una de las competencias fijadas por la Comisión Europea, aprender a aprender (Gargallo, Pérez-Pérez, García-García, Giménez y Portillo, 2020). El factor Ayuda indica que el alumno pide la asistencia a los compañeros o al profesor para la realización de las actividades académicas. Como indican Vázquez, Noriega y García (2013) promover estrategias de aprendizaje donde se pida ayuda al profesor, compañeros o los referentes académicos es un indicador de buen rendimiento final. Daura (2015) plantea que hay que acompañar a los estudiantes durante su periodo de formación.

Considerando los siete factores que se analizan en relación a establecer alguna diferencia en las estrategias de aprendizaje respecto a los estudiantes universitarios que están trabajando y los que no trabajan, existen diferencia significativa en cinco de ellos: Elaboración, Aprovechamiento del tiempo-concentración, Ayuda, Constancia y Autointerrogación. Para ambos grupos de la muestra, como se puede observar en la figuras 1, 2 y 3, valoran de manera que "le describe suficientemente" los factores Elaboración, Metacognición y Organización. Para la Elaboración, se enfatiza en que los estudiantes deben hacer uso de la lectura para asociar la información que están obteniendo con la que ya poseían, buscando a su vez asimilar adecuadamente el contenido de la asignatura. En el factor Metacognición, indicado para concretar las metas, ajustando el estudio y la asimilación de información, los universitarios deben ser capaces de reflexionar para captar la esencia de los conocimientos que tienen que adquirir. Les lleva previamente a revisar el material y entender cómo está organizado. Como indican Sáiz y Valdivieso-León (2020) para la formación superior de los futuros profesionales es necesario que haya una percepción ajustada entre los conocimientos y las habilidades necesarias. En la Organización, resulta significativo las estrategias utilizadas en la adquisición de conocimiento, como por ejemplo, subrayar apuntes, acceder a lecturas para ampliar conceptos relevantes, etc. (García y Jiménez, 2014). Otros estudios (Marcén y Martínez-Caraballo, 2011) demuestran que menos del $4 \%$ de los universitarios poseen un plan de trabajo bien definido. Este tipo de hábitos les lleva a una 
mala distribución del tiempo, concentrándose en la época de exámenes y cuando deben entregar actividades. Un correcto grado de organización es un buen discriminante en los factores académicos (Tomás, Expósito y Sempere, 2014) así como promover el interés por aprender por parte del docente (Cáceres, Muñoz y Valenzuela, 2021). Destaca en el factor Autointerrogación, la diferencia que se produce entre la muestra de estudiantes que trabajan y los que no trabajan. Para finalizar su proceso de reflexión no basta con conocer en qué momento estudian la materia, sino que es el profesor quien debe guiarlos en desarrollarla, cerciorarse de que la han entendido y resolver sus dudas (Casanellas et al., 2014).

Como conclusión general, los estudiantes valoran como que "no están seguros" en los factores Ayuda, Constancia y Aprovechamiento del tiempo-Concentración. En relación a la Ayuda, los estudiantes deben ser capaces de solicitar a sus compañeros colaboración en las actividades que no comprendan. En el factor Constancia, para realizar satisfactoriamente las diferentes actividades y trabajos, los alumnos confían en que la asistencia a clase regular y leer las notas que tomaron en las clases les beneficia como estrategia de aprendizaje. Diferentes investigaciones (García, Alvarado y Jiménez, 2000; Rodríguez y Herrera, 2009, p.2) que llevaron a cabo un análisis sobre regresión logística, concluyeron que "la asistencia y participación en clase son variables que predicen el rendimiento académico universitario". Finalmente, el factor Aprovechamiento del tiempo-Concentración, recoge información sobre cómo gestiona el tiempo y la disposición a centrarse en la actividad que está realizando. Esta búsqueda de condiciones ambientales fue una de las estrategias individuales más utilizadas (García y Jiménez, 2014). Los estudiantes universitarios deben disponer de un lugar fijo de estudio. La figura del profesor debe servir para que el estudiante obtenga el mayor provecho de sus capacidades, gestionándolas adecuadamente (Roces, Tourón y González, 1995; Salinas, De Benito y Lizana, 2014). El EEES nos plantea el rol del docente, guía en la adquisición de conocimiento de los estudiantes, siendo este el protagonista de su formación (Olmedo, 2013) y donde el docente puede promover el éxito educativo en sus estudiantes (Boroel, Sánchez, Morales y Henríquez, 2018), también haciéndoles partícipes del proceso de evaluación (Gárgano, 2020 y Abella, Ausín, Delgado y Casado, 2020).

Una de las medidas que se pueden aplicar para optimizar los recursos de los estudiantes durante su formación, es el uso y gestión de las TIC. Teniendo en cuenta cifras recientemente publicadas sobre el tiempo que dedican los estudiantes universitarios a las TIC, el 58,3\% de los estudiantes dedican más de cinco horas diarias al teléfono móvil (Garrote-Rojas, JiménezFernández y Gómez-Barreto, 2018). Los estudiantes debe disponer de la tecnología necesaria para su formación, evitando así ampliar la brecha digital (Cabero y Valencia, 2020).

Teniendo en cuenta la importancia de poseer unas adecuadas estrategias de estudio durante la formación, en futuras investigaciones podrían considerarse las puntuaciones obtenidas en las asignaturas para determinar qué impacto tienen las estrategias en los resultados alcanzados.

\section{Referencias}

Abella, V., Ausín, V., Delgado, V., \& Casado, R. (2020). Aprendizaje basado en proyectos y estrategias de evaluación formativas: percepción de los estudiantes universitarios. Revista Iberoamericana de Evaluación Educativa, 13(1), 93-110. Doi: 10.15366/riee2020.13.1.004

Abellán, H., \& Herrada, R.I. (2016). Innovación educativa y metodologías activas en educación secundaria: la perspectiva de los docentes de lengua castellana y literatura. Revista Fuentes, 18(1), 65-76. doi: 10.12795/revistafuentes.2016.i18.04 
Black, P., \& Wiliam, D. (1998). Assessment and classroom learning. Assessment in Education: Principles, Policy \& Practice, 5(1), 7-74.

Boroel, B. I., Sánchez, J., Morales K.D., \& Henríquez, P.S. (2018). Educación exitosa para todos: la tutoría como proceso de acompañamiento escolar desde la mirada de la equidad educativa. Revista Fuentes, 20(2), 91-104.

Boza, A., \& Méndez, A.M. (2013). Aprendizaje motivado en alumnos universitarios: validación y resultados generales de una escala. Revista de Investigación Educativa, 31(2), 331-347.

Boza, A., \& Toscana M.O. (2012). Motivos, actitudes y estrategias de aprendizaje: aprendizaje motivado en alumnos universitarios. Profesorado: Revista de currículum y formación del profesorado, 16(1), 125-142

Brookhart, S.M. (2007). Expanding views about formative classroom assessment: A review of the literature. En J. H. McMillan (ed.), Formative classroom assessment: Research, theory and practice (pp. 43-62). New York: Teachers College Press.

Cabero, J., \& Valencia, R. (2020). Y el COVID-19 transformó el sistema educativo: reflexiones y experiencias por aprender. Revista Internacional de Investigación e Innovación Educativa, 15, 218-228. Doi: 10.46661/ijeri.5246

Cáceres, C., Muñoz, C., \& Valenzuela, J. (2021). Responsabilidad personal docente y motivación escolar. Revista Electrónica Interuniversitaria de Formación del Profesorado, 24(1), 175-188. Doi: 10.6018/reifop.402761

Camero, F., Martín, F., \& Herrero, J. (2000). Estilos y estrategias de aprendizaje en estudiantes universitarios. Psicothema, 12(4), 615-622.

Cano, E.I., Chamizo, J., \& Valiente, C. (2014). Los resultados de aprendizaje como indicadores de mejora continua y herramienta de gobernanza. Los estudios de Contabilidad y Finanzas en la London South Bank University (Reino Unido). Revista de Formación e Innovación Educativa Universitaria, 7(1), 59-74.

Casanellas, M., Camós, M., Medir, LL., Momtolio, D., Sayós, R., \& Solé, M. (2014). Instrumentos para la evaluación de la competencia transversal de capacidad de aprendizaje. Depósito Digital: Universidad de Barcelona. Recuperado de http://hdl.handle.net/2445/54508

Cuadrado, I., \& Fernández, I. (2008). Nuevas competencias del profesor en el EEES: Una experiencia de innovación docente. Teoría de la Educación: Educación y Cultura en la Sociedad de la Información, 9(1 rendimiento académico en estudiantes del ciclo clínico de la carrera de Medicina. Revista electrónica de investigación educativa, 17(3), 28-45.

Delgado, A.M. (Coord.) (2006). Evaluación de las competencias en el Espacio Europeo de Educación Superior. Barcelona: Bosch Editor.

Eccles, J., Adler T.F., Futterman, R., Goff, S.B., \& Kaczala, C.M. (1983). Expectancies, values, and academic behaviors. En J.T. Spence (Ed.), Achievement and achievement motivation (pp. 75-146). San Francisco, CA: Freeman.

Ferguson, P. (2011). Student perceptions of quality feedback in teacher education. Assessment and Evaluation in Higher Education, 36(1), 51- 62.

Fraile, A., López-Pastor, V., Castejón, J., \& Romero, R. (2013). La evaluación formativa en docencia universitaria y el rendimiento académico del alumnado. Aula abierta, 41(2). 23-34. 
García, M.V., Alvarado, J.M., \& Jiménez, A. (2000). La predicción del rendimiento académico: regresión lineal versus regresión logística. Psicothema, 12(2), 248-252.

García, M.M., \& Jiménez, A. (2014). Estrategias en el aprendizaje de la lengua extranjera y niveles de competencia en estudiantes universitarios de magisterio. Revista de investigación Educativa, 32(2), 363-378.

Gargallo,B., Pérez-Pérez, C., García-García, F.J., Giménez, J.A. \& Portillo, N. (2020). La competencia aprender a aprender en la universidad: propuesta de modelo teórico. Educación XX1, 23(1), 19-44. Doi: 10.5944/educXX1.23367

Garrote-Rojas, D., Jiménez-Fernández, S., \& Gómez-Barreto, I.M. (2018). Problemas derivados del uso de internet y el teléfono móvil en estudiantes universitarios. Formación Universitaria, 11(2), 99-108.

Garrote, D., Garrote, C., \& Jiménez, S. (2016). Factores influyentes en motivación y estrategias de aprendizaje en los alumnos de grado. Revista Iberoamericana sobre Calidad, Eficacia y Cambio en Educación, 14(2), 31-44.

Gárgano, S. (2020). Análisis de las estrategias de evaluación de los aprendizajes de las materias de filosofía humana y aplacada de las carreras de educación física. Revista Electrónica Interuniversitaria de Formación del Profesorado, 23(3), 201-215. Doi: 10.6018/reifop.366371

Goñi, J. M. (2005). El Espacio Europeo de Educación Superior, un reto para la universidad. Barcelona: Octaedro/ICE Universidad de Barcelona.

Hernández-Pina, F., Clares, P., Rosario, P., \& Espin, M. (2005). Aprendizaje, competencias y rendimiento en Educación Superior. La Muralla: Madrid.

Herrera, L., Lorenzo, O., \& Rodríguez, C. (2008). Las tutorías en el Espacio Europeo de Educación Superior: valoración de su implementación en la titulación de maestro. Revista de Investigación Psicoeducativa, 6(1), 65-85.

Kolb, D. A. (1984): Experiential Learning. Experience as the source of learning and development. Englewood Cliffs, N.J.: Prentice-Hall, Inc.

Malbernat, L.R. (2008). Cambios institucionales para una nueva enseñanza en la educación superior. Profesorado, Revista de Currículo y Formación del Profesorado, 12(2), 1-18.

Martínez-Fernández, J. R., \& Galán, F. (2000). Estrategias de aprendizaje, motivación y rendimiento académico en alumnos universitarios. Revista Española de Orientación y Psicopedagogía, 11(19), 35-50.

Monereo, C., \& Badia, A. (2013). Aprendizaje estratégico y tecnologías de la información y la comunicación: una revisión crítica. Revista teoría de la Educación: Educación y Cultura en la Sociedad de la Información, 14(2), 15-41.

Moreno, S., Bajo, M. T., Moya, M., Maldonado, A., \& Tudela, P. (2007). Las competencias en el nuevo paradigma educativo para Europa. Granada: Vicerrectorado de Planificación, Calidad y Evaluación Docente de la Universidad de Granada.

Nicol, D., \& Macfarlane, D. (2006). Formati- ve assessment and self-regulated learning: A model and seven principles of good feedback practice. Studies in Higher Education, 31(2), 199-218.

Oliveros, L. (2006). Identificación de competencias: una estrategia para la formación en el Espacio Europeo de Educación Superior. Revista Complutense de Educación, 17(1), 101118. 
Olmedo, E. M. (2013). Enfoques de aprendizaje de los estudiantes y metodología docente: Evolución hacia el nuevo sistema de formación e interacción propuesta en el EEES. Revista de Investigación Educativa, 31(2), 411-429.

Palomares A., \& Garrote, D. (2009). Un nuevo modelo docente por y para el alumnado. Revista Ensayos, 24, 25-34.

Pintrich, P., \& Degroot, E. (1990). Motivational and self-regulated learning components of class- room academic performance. Journal of Educational Psychology, 82(1), 33-40.

Pintrich, P., Mckeachi, W., Smith, D., Doljanac, R., Lin, Y., Naveh-Benjamin, M., Crooks, T. y Karabenick, S. (1988). Motivated strategies for learning questionnaire. Michigan: The University of Michigan (NCRIPTAL).

Pintrich, P., Smith, D., García, T., \& Mckeachie, W. (1993). Reliability and predictive validity of the motivated strategies for learning questionnaire (MSLQ). Educational and Psychological Measurement, 53, 801-813.

Roces, C., Tourón, J., \& González, M. C. (1995). Validación preliminar del CEAM II (Cuestionario de estrategias de aprendizaje y motivación II). Psicología, 16(3), 347-366.

Rodríguez , C., \& Herrera, L. (2009). Análisis correlacional-predictivo de la influencia de la asistencia a clase en el rendimiento académico universitario. Estudio de caso en una asignatura. Profesorado: revista de currículum y formación del profesorado, 13(2), 1-13.

Sadler, D. R. (2010). Beyond feedback: Developing student capability in complex appraisal. Assessment and evaluation in higher education, 35(5), 535-550.

Sáiz, M.C., \& Valdivieso-León, L. (2020). Relación entre rendimientos académico y desarrollo de Estrategias de autorregulación en estudiantes universitarios. Revista Electrónica Interuniversitaria de Formación del Profesorado, 23(3), 49-65. Doi: 10.6018/reifop.385491

Salinas, J., De Benito, B., \& Lizana, A. (2014). Competencias docentes para los nuevos escenarios de aprendizaje. Revista Interuniversitaria de Formación del Profesorado, 79(28.1), 145-163.

Tomás, J.V., Expósito, M., \& Sempere, S. (2014). Determinantes del rendimiento académico en los estudiantes de grado. Un estudio en administración y dirección de empresas. Revista de Investigación Educativa, 32(2), 379-392.

Tomusk, V. (2006). Creating the European Area of Higher Education: Voices from the periphery. Dordrecht: Springer.

Vázquez, E., \& Sevillano, M. (2015). Dispositivos digitales móviles en educación. El aprendizaje ubicuo. Madrid: Narcea.

Vázquez, S., Noriega, M. y García, S. (2013). Relaciones entre rendimiento académico, competencia espacial, estilos de aprendizaje y deserción. Revista Electrónica de Investigación Educativa, 15(1), 29-44.

Yorke, M. (2003). Formative assessment in higher education: Moves towards theory and the enhancement of pedagogic practice. Higher Education, 45(4), 477-501.

Zabalza, M.A. (2003). Competencias docentes del profesorado universitario. Calidad y desarrollo profesional. Madrid: Narcea. 